\title{
THREE-DIMENSIONAL SHARP HARDY-LERAY INEQUALITY FOR SOLENOIDAL FIELDS
}

\section{NAOKI HAMAMOTO}

\begin{tabular}{|c|l|}
\hline Citation & OCAMI Preprint Series \\
\hline Issue Date & 2019 \\
\hline Type & Preprint \\
\hline Textversion & Author \\
\hline Rights & For personal use only. No other uses without permission. \\
\hline Relation & $\begin{array}{l}\text { The following article has been submitted to Nonlinear Analysis. After it is } \\
\text { published, it will be found at https://doi.org/10.1016/j.na.2019.111634 }\end{array}$ \\
\hline
\end{tabular}

From: Osaka City University Advanced Mathematical Institute http://www.sci.osaka-cu.ac.jp/OCAMI/publication/preprint/preprint.html 


\title{
THREE-DIMENSIONAL SHARP HARDY-LERAY INEQUALITY FOR SOLENOIDAL FIELDS
}

\author{
NAOKI HAMAMOTO
}

\begin{abstract}
This is a complement of the former works by Costin-Maz'ya [1] and HamamotoTakahashi [5] on sharp Hardy-Leray inequality for solenoidal (i.e., divergence-free) fields in $\mathbb{R}^{3}$ with some axisymmetry conditions. Here we derive the same best constant, with no assumption of any symmetry.
\end{abstract}

\section{INTRODUCTION}

Let $N \geq 3$ be an integer. Throughout this paper, we use bold letters to denote $N$ dimensional vectors, e.g., $\boldsymbol{x}=\left(x_{1}, x_{2}, \cdots, x_{N}\right) \in \mathbb{R}^{N}$. In the following, $C_{c}^{\infty}\left(\mathbb{R}^{N}\right)^{N}$ denotes the space of all smooth vector fields

$$
\boldsymbol{u}=\left(u_{1}, u_{2}, \cdots, u_{N}\right): \mathbb{R}^{N} \rightarrow \mathbb{R}^{N}, \quad \boldsymbol{x} \mapsto \boldsymbol{u}(\boldsymbol{x})=\left(u_{1}(\boldsymbol{x}), u_{2}(\boldsymbol{x}), \cdots, u_{N}(\boldsymbol{x})\right)
$$

with compact support on $\mathbb{R}^{N}$.

Let $\gamma \in \mathbb{R}$ be a real number. The Hardy-Leray inequality with weight $\gamma$ is given by

$$
C_{N, \gamma} \int_{\mathbb{R}^{N}} \frac{|\boldsymbol{u}|^{2}}{|\boldsymbol{x}|^{2}}|\boldsymbol{x}|^{2 \gamma} d x \leq \int_{\mathbb{R}^{N}}|\nabla \boldsymbol{u}|^{2}|\boldsymbol{x}|^{2 \gamma} d x
$$

for all $\boldsymbol{u} \in C_{c}^{\infty}\left(\mathbb{R}^{N}\right)^{N}$ such that $\boldsymbol{u}(\mathbf{0})=\mathbf{0}$ if $\gamma \leq 1-N / 2$, where the constant number $C_{N, \gamma}=\left(\gamma+\frac{N}{2}-1\right)^{2}$ is sharp. J. Leray [8] proved the inequality (1.1) for $N=3$ and $\gamma=0$ in his works on the Navier-Stokes equations, as a generalization of the one-dimensional inequality by G. H. Hardy [6]. We are interested in the problem whether $C_{N, \gamma}$ in (1.1) is improved to be larger if the selection of $\boldsymbol{u}$ is restricted to a smaller subspace of $C_{c}^{\infty}\left(\mathbb{R}^{N}\right)^{N}$. This problem has been answered in some cases or variations: Taking into account the context of hydrodynamics, O. Costin and V. Maz'ya [1] derived a new sharp value of $C_{N, \gamma}$ by assuming $\boldsymbol{u}$ to be solenoidal (i.e., divergence-free) and axisymmetric. After a decade, N. Hamamoto and F. Takahashi [4] computed another sharp value of $C_{N, \gamma}$ in the case where $\boldsymbol{u}$ is conservative; this computation was done with no use of any symmetry condition. At present, the case of solenoidal fields still remains unanswered under no assumption of any symmetry, which is the main theme of this paper. Incidentally, it should be pointed out that the sharpness of Rellich-Leray inequality (which is a second-order version of Hardy-Leray inequality) was also studied for axisymmetric solenoidal fields or conservative fields $[3,4]$.

Hereafter we restrict ourselves to the case $N=3$. The three-dimensional version of the preceding result [1] reads as follows:

Theorem 1 (O. Costin and V. Maz'ya [1]). Let $\boldsymbol{u} \in C_{c}^{\infty}\left(\mathbb{R}^{3}\right)^{3}$ be an axisymmetric solenoidal field such that $\boldsymbol{u}(\mathbf{0})=\mathbf{0}$ for $\gamma \leq-1 / 2$. Then inequality

$$
\begin{aligned}
& \qquad C_{\gamma} \int_{\mathbb{R}^{3}} \frac{|\boldsymbol{u}|^{2}}{|\boldsymbol{x}|^{2}}|\boldsymbol{x}|^{2 \gamma} d x \leq \int_{\mathbb{R}^{3}}|\nabla \boldsymbol{u}|^{2}|\boldsymbol{x}|^{2 \gamma} d x \\
& \text { holds with the best constant } C_{\gamma}= \begin{cases}\left(\gamma+\frac{1}{2}\right)^{2} \frac{4+\left(\gamma-\frac{3}{2}\right)^{2}}{2+\left(\gamma-\frac{3}{2}\right)^{2}}, & \text { for } \gamma \leq 1, \\
\left(\gamma+\frac{1}{2}\right)^{2}+2, & \text { for } \gamma>1 .\end{cases}
\end{aligned}
$$

2010 Mathematics Subject Classification. Primary 35A23; Secondary 26D10.

Key words and phrases. Hardy-Leray inequality, solenoidal, poloidal, toroidal, Laplace-Beltrami operator. 
As a slight refinement of this theorem, N. Hamamoto and F. Takahashi [5] showed that the axisymmetry condition on $\boldsymbol{u}$ can be relaxed to that on only the swirl (or azimuthal) component of $\boldsymbol{u}$, without changing the value of $C_{\gamma}$. This time, we will further completely remove such a symmetry condition. Now our main result is the following:

Theorem 2. Let $\boldsymbol{u} \in C_{c}^{\infty}\left(\mathbb{R}^{3}\right)^{3}$ be a solenoidal field such that $\boldsymbol{u}(\mathbf{0})=\mathbf{0}$ for $\gamma \leq-1 / 2$. Then inequality

$$
C_{\gamma} \int_{\mathbb{R}^{3}} \frac{|\boldsymbol{u}|^{2}}{|\boldsymbol{x}|^{2}}|\boldsymbol{x}|^{2 \gamma} d x \leq \int_{\mathbb{R}^{3}}|\nabla \boldsymbol{u}|^{2}|\boldsymbol{x}|^{2 \gamma} d x
$$

holds with the same constant $C_{\gamma}$ as in Theorem 1.

In the setting of spherical coordinates (in $\S 2$ ), the previous Theorem 1 was proved in [1] by choosing latitudinal and azimuthal components of $\boldsymbol{u}$ as two independent test functions. Theorem 2 is, however, difficult to prove in the same way, roughly because the two angular components are not necessarily $L^{2}$-orthogonal unless $\boldsymbol{u}$ is axisymmetric. To overcome such a difficulty, we choose the so-called poloidal-toroidal potentials (in §3) as alternative test functions. After these preparations, the proof of Theorem 2 will be given in $\S 4$.

\section{First Preparation: SPherical COORDinates}

In this section, we introduce a spherical coordinate system and derive some useful formulae for differential operators on the sphere

$$
\mathbb{S}^{2}=\left\{\boldsymbol{x} \in \mathbb{R}^{3} ; x_{1}^{2}+x_{2}^{2}+x_{3}^{2}=1\right\} .
$$

The spherical coordinate system in $\mathbb{R}^{3}$ is composed of the radius $\rho>0$ and the two angles: colatitude $\theta \in[0, \pi]$ and azimuth $\varphi \in[0,2 \pi)$. The position of a vector $\boldsymbol{x}=\left(x_{1}, x_{2}, x_{3}\right) \in \mathbb{R}^{3}$ is then represented by $\boldsymbol{x}=\rho \boldsymbol{\sigma}$, where $\boldsymbol{\sigma}=\boldsymbol{\sigma}(\theta, \varphi) \in \mathbb{S}^{2}$ together with the orthonormal basis $R(\boldsymbol{\sigma})=\left(\boldsymbol{\sigma}, \boldsymbol{e}_{\theta}, \boldsymbol{e}_{\varphi}\right) \in S O(3)$ given by

$$
\left\{\begin{array}{l}
\boldsymbol{\sigma}=(\cos \theta, \sin \theta \cos \varphi, \sin \theta \sin \varphi) \\
\boldsymbol{e}_{\theta}=\partial_{\theta} \boldsymbol{\sigma}=(-\sin \theta, \cos \theta \cos \varphi, \cos \theta \sin \varphi) \\
\boldsymbol{e}_{\varphi}=\partial_{\varphi} \boldsymbol{\sigma}=(0,-\sin \varphi, \cos \varphi) .
\end{array}\right.
$$

Hereafter we write the partial derivatives simply as $\partial_{\theta}=\frac{\partial}{\partial \theta}, \partial_{\varphi}=\frac{\partial}{\partial \varphi}$, etc. Also we use the abbreviation

$$
\partial_{\varphi}:=\frac{1}{\sin \theta} \partial_{\varphi}
$$

By differentiating $\boldsymbol{e}_{\theta}$ and $\boldsymbol{e}_{\varphi}$, we can directly verify that

$$
\begin{cases}\partial_{\theta} \boldsymbol{e}_{\theta}=-\boldsymbol{\sigma}, & \partial_{\varphi} \boldsymbol{e}_{\theta}=\boldsymbol{e}_{\varphi} \cot \theta \\ \partial_{\theta} \boldsymbol{e}_{\varphi}=\mathbf{0}, & \partial_{\varphi} \boldsymbol{e}_{\varphi}=-\boldsymbol{\sigma}-\boldsymbol{e}_{\theta} \cot \theta\end{cases}
$$

Now let $\boldsymbol{u}=\left(u_{1}, u_{2}, u_{3}\right): \mathbb{R}^{3} \rightarrow \mathbb{R}^{3}$ be a vector field. Then its radial and angular components $u_{\rho}, u_{\theta}, u_{\varphi}$ are defined as the expansion coefficients of $\boldsymbol{u}$ in the basis $R(\boldsymbol{\sigma})$ :

$$
\boldsymbol{u}(\boldsymbol{x})=\boldsymbol{u}(\rho \boldsymbol{\sigma})=\boldsymbol{\sigma} u_{\rho}+\boldsymbol{e}_{\theta} u_{\theta}+\boldsymbol{e}_{\varphi} u_{\varphi}
$$

Also let us expand the gradient operator $\nabla=\left(\frac{\partial}{\partial x_{1}}, \frac{\partial}{\partial x_{2}}, \frac{\partial}{\partial x_{3}}\right)$ in the same basis: by the differential chain rule together with $(2.1)$, we have

$$
\begin{aligned}
& \partial_{\rho}=\partial_{\rho} \boldsymbol{x} \cdot \nabla=\boldsymbol{\sigma} \cdot \nabla, \\
& \partial_{\theta}=\partial_{\theta} \boldsymbol{x} \cdot \nabla=\rho \boldsymbol{e}_{\theta} \cdot \nabla, \\
& \partial_{\varphi}=\partial_{\varphi} \boldsymbol{x} \cdot \nabla=(\rho \sin \theta) \boldsymbol{e}_{\varphi} \cdot \nabla,
\end{aligned}
$$

where "." denotes the standard inner product in $\mathbb{R}^{3}$. Then it turns out that

$$
\nabla=\boldsymbol{\sigma} \partial_{\rho}+\frac{1}{\rho} \nabla_{\sigma}
$$


where $\nabla_{\sigma}=\boldsymbol{e}_{\theta} \partial_{\theta}+\boldsymbol{e}_{\varphi} \theta_{\varphi}$ is the gradient operator on $\mathbb{S}^{2}$. When they act on $\boldsymbol{x}=\rho \boldsymbol{\sigma}$ or $\rho=|\boldsymbol{x}|$, we verify the formulae

$$
\begin{aligned}
& \boldsymbol{y} \cdot \nabla \boldsymbol{x}=\boldsymbol{y}, \quad \nabla \rho=\boldsymbol{\sigma} \\
& \boldsymbol{y} \cdot \nabla_{\sigma} \boldsymbol{\sigma}=\boldsymbol{y}-(\boldsymbol{y} \cdot \boldsymbol{\sigma}) \boldsymbol{\sigma}
\end{aligned}
$$

for all $\boldsymbol{y} \in \mathbb{R}^{3}$. Indeed, the first line follows by direct differential calculation in the standard $\mathbb{R}^{3}$ coordinates, while the last equality follows from (2.4):

$$
\begin{aligned}
\boldsymbol{y} \cdot \nabla_{\sigma} \boldsymbol{\sigma} & =\boldsymbol{y} \cdot \rho \nabla \boldsymbol{\sigma}=\boldsymbol{y} \cdot \rho \nabla \frac{\boldsymbol{x}}{\rho}=\boldsymbol{y} \cdot \frac{(\nabla \boldsymbol{x}) \rho-\boldsymbol{x} \nabla \rho}{\rho} \\
& =\boldsymbol{y} \cdot \nabla \boldsymbol{x}-\boldsymbol{y} \cdot \boldsymbol{\sigma} \boldsymbol{\sigma}=\boldsymbol{y}-(\boldsymbol{y} \cdot \boldsymbol{\sigma}) \boldsymbol{\sigma} .
\end{aligned}
$$

The Laplace operator $\Delta=\nabla \cdot \nabla=\sum_{k=1}^{3}\left(\frac{\partial}{\partial x_{k}}\right)^{2}$ is known to be written in terms of the spherical coordinates as

$$
\Delta=\frac{1}{\rho^{2}} \partial_{\rho}\left(\rho^{2} \partial_{\rho}\right)+\frac{1}{\rho^{2}} \Delta_{\sigma}
$$

where $\Delta_{\sigma}=D_{\theta} \partial_{\theta}+\partial_{\varphi}^{2}$ is the Laplace-Beltrami operator on $\mathbb{S}^{2} ;$ the operator $D_{\theta}=\partial_{\theta}+\cot \theta$ is adjoint to $-\partial_{\theta}$ in $L^{2}\left(\mathbb{S}^{2}\right)$ :

$$
-\int_{\mathbb{S}^{2}}\left(\partial_{\theta} f\right) g d \sigma=\int_{\mathbb{S}^{2}} f D_{\theta} g d \sigma, \quad d \sigma=\sin \theta d \theta d \varphi
$$

for any $f, g \in C^{\infty}\left(\mathbb{S}^{2}\right)$.

For later use, we give the following technical lemma:

Lemma 3. Let $f: \mathbb{S}^{2} \rightarrow \mathbb{R}$ be a smooth function of the variable $\boldsymbol{\sigma} \in \mathbb{S}^{2}$. Then

$$
\begin{aligned}
& \Delta_{\sigma}(\boldsymbol{\sigma} f)-\boldsymbol{\sigma} \Delta_{\sigma} f=2\left(\nabla_{\sigma} f-\boldsymbol{\sigma} f\right), \\
& \Delta_{\sigma} \nabla_{\sigma} f-\nabla_{\sigma} \Delta_{\sigma} f=-2 \boldsymbol{\sigma} \Delta_{\sigma} f .
\end{aligned}
$$

proof. Let us identify $f \in C^{\infty}\left(\mathbb{S}^{2}\right)$ with $\widetilde{f} \in C^{\infty}\left(\mathbb{R}^{3} \backslash\{\mathbf{0}\}\right)$ by the relation $\widetilde{f}(\boldsymbol{x})=f(\boldsymbol{x} /|\boldsymbol{x}|)$. The key idea of the proof is to express the operators on $\mathbb{S}^{2}$ in terms of the standard $\mathbb{R}^{3}$ coordinates in which the derivatives are commutative: $\Delta \nabla=\nabla \Delta$. By using the Leibniz rule and $(2.4),(2.6),(2.5)$ for $\boldsymbol{y}=\nabla_{\sigma} f$, we see

$$
\begin{aligned}
\Delta_{\sigma}(\boldsymbol{\sigma} f)-\boldsymbol{\sigma} \Delta_{\sigma} f & =\left(\Delta_{\sigma} \boldsymbol{\sigma}\right) f+2 \nabla_{\sigma} f \cdot \nabla_{\sigma} \boldsymbol{\sigma} \\
& =\left(\rho^{2} \Delta \nabla \rho\right) f+2\left(\nabla_{\sigma} f-\left(\left(\nabla_{\sigma} f\right) \cdot \boldsymbol{\sigma}\right) \boldsymbol{\sigma}\right) \\
& =\left(\rho^{2} \nabla \Delta \rho\right) f+2 \nabla_{\sigma} f=\left(\rho^{2} \nabla \frac{2}{\rho}\right) f+2 \nabla_{\sigma} f \\
& =-2(\nabla \rho) f+2 \nabla_{\sigma} f=-2 \boldsymbol{\sigma} f+2 \nabla_{\sigma} f
\end{aligned}
$$

which proves the first line of (2.7). The proof of the second line also follows similarly:

$$
\begin{aligned}
\left(\Delta_{\sigma} \nabla_{\sigma}-\nabla_{\sigma} \Delta_{\sigma}\right) f & =\rho^{2} \Delta \nabla_{\sigma} f-\rho \nabla \Delta_{\sigma} f \\
& =\rho^{2} \Delta(\rho \nabla f)-\rho \nabla\left(\rho^{2} \Delta f\right) \\
& =\rho^{2}((\Delta \rho) \nabla f+2 \nabla \rho \cdot \nabla \nabla f)-\rho\left(\nabla \rho^{2}\right) \Delta f \\
& =2 \rho \nabla f+2 \rho^{2} \boldsymbol{\sigma} \cdot \nabla \nabla f-2 \rho^{2}(\nabla \rho) \Delta f \\
& =2 \nabla_{\sigma} f+2 \rho^{2} \partial_{\rho} \frac{1}{\rho} \nabla_{\sigma} f-2 \boldsymbol{\sigma} \Delta_{\sigma} f \\
& =-2 \boldsymbol{\sigma} \Delta_{\sigma} f .
\end{aligned}
$$




\section{Second Preperation: Poloidal-Toroidal Representation of solenoidal} FIELDS

Let us define $G$ as a linear operator, that maps every solenoidal field $\boldsymbol{u} \in C_{c}^{\infty}\left(\mathbb{R}^{3}\right)^{3}$ to a continuous function $G \boldsymbol{u}: \mathbb{R}^{3} \rightarrow \mathbb{R}$, by the formula

$$
G \boldsymbol{u}(\boldsymbol{x})=\frac{|\boldsymbol{x}|}{4 \pi} \int_{\mathbb{S}^{2}} \boldsymbol{\sigma}^{\prime} \cdot \boldsymbol{u}\left(|\boldsymbol{x}| \boldsymbol{\sigma}^{\prime}\right) \log \left(1-\frac{\boldsymbol{x}}{|\boldsymbol{x}|} \cdot \boldsymbol{\sigma}^{\prime}\right) d \sigma^{\prime} \quad \text { for all } \quad \boldsymbol{x} \in \mathbb{R}^{3} \backslash\{\mathbf{0}\} .
$$

This function is called the poloidal potential of $\boldsymbol{u}$. Then $G$ also maps the rotation of $\boldsymbol{u}$,

$$
\nabla \times \boldsymbol{u}=\left(\partial_{x_{2}} u_{3}-\partial_{x_{3}} u_{2}, \partial_{x_{3}} u_{1}-\partial_{x_{1}} u_{3}, \partial_{x_{1}} u_{2}-\partial_{x_{2}} u_{1}\right)
$$

to the function $G(\nabla \times \boldsymbol{u})$ which is called the toroidal potential of $\boldsymbol{u}$. The logarithmic function $\log \left(1-\boldsymbol{\sigma} \cdot \boldsymbol{\sigma}^{\prime}\right)$ in the right-hand side of (3.1) appears in e.g. [2, V§11.4] or [7], as a Green's function of the Laplace-Beltrami operator on $\mathbb{S}^{2}$; this enables $G \boldsymbol{u}$ to satisfy the Poisson-Beltrami equation:

$$
\Delta_{\sigma} G \boldsymbol{u}=\boldsymbol{x} \cdot \boldsymbol{u} \quad \text { in } \mathbb{R}^{3} \backslash\{\mathbf{0}\}
$$

Although this is well-known, we give its proof in Appendix in our style of notation, for the convenience of the readers.

Also let us introduce the derivative operator

$$
\boldsymbol{J}:=\boldsymbol{x} \times \nabla=\boldsymbol{\sigma} \times \nabla_{\sigma}=\boldsymbol{e}_{\varphi} \partial_{\theta}-\boldsymbol{e}_{\theta} \partial_{\varphi},
$$

which stands for an angular momentum in quantum mechanics. Note that $\boldsymbol{J}$ commutes with both of $\Delta$ and $\Delta_{\sigma}$ :

$$
\Delta \boldsymbol{J}=\boldsymbol{J} \Delta, \quad \Delta_{\sigma} \boldsymbol{J}=\boldsymbol{J} \Delta_{\sigma} ;
$$

the former equality is easy to check by using the standard coordinates, whereas the latter follows from the former by use of (2.6).

The following proposition is known as the so-called poloidal-toroidal decomposition of solenoidal fields:

Proposition 4. Let $\boldsymbol{u} \in C_{c}^{\infty}\left(\mathbb{R}^{3}\right)^{3}$ be a solenoidal field. Let $A, B$ denote the poloidal, toroidal potentials of $\boldsymbol{u}$, that is, $A=G \boldsymbol{u}, B=G(\nabla \times \boldsymbol{u})$. Then they satisfy the PoissonBeltrami equations

$$
\Delta_{\sigma} A=\boldsymbol{x} \cdot \boldsymbol{u}, \quad \Delta_{\sigma} B=\boldsymbol{x} \cdot(\nabla \times \boldsymbol{u})
$$

and the poloidal-toroidal decomposition formula

$$
\boldsymbol{u}=\boldsymbol{u}_{P}+\boldsymbol{u}_{T}, \quad\left\{\begin{array}{l}
\boldsymbol{u}_{P}:=\nabla \times \boldsymbol{J} A=\boldsymbol{\sigma} \rho^{-1} \Delta_{\sigma} A-\left(\partial_{\rho}+\frac{1}{\rho}\right) \nabla_{\sigma} A \\
\boldsymbol{u}_{T}:=\boldsymbol{J} B
\end{array}\right.
$$

for all $\boldsymbol{x}=\rho \boldsymbol{\sigma} \neq \mathbf{0}$. Moreover, this is an $L^{2}$-orthogonal decomposition in the sense that

$$
\int_{\mathbb{S}^{2}} \boldsymbol{u}_{P}(\rho \boldsymbol{\sigma}) \cdot \boldsymbol{u}_{T}(\rho \boldsymbol{\sigma})=\int_{\mathbb{S}^{2}} \nabla \boldsymbol{u}_{P}(\rho \boldsymbol{\sigma}) \cdot \nabla \boldsymbol{u}_{T}(\rho \boldsymbol{\sigma}) d \sigma=0
$$

proof. The proof of the equations in (3.2) follows by using Lemma 5 in Appendix.

We now prove (3.3). As in [9], it suffices to check that the solenoidal field

$$
\boldsymbol{w}(\boldsymbol{x}):=\boldsymbol{u}(\boldsymbol{x})-\nabla \times \boldsymbol{J} A-\boldsymbol{J} B
$$


vanishes everywhere; to do so, by direct calculation of the last two terms and their rotations, we verify that:

$$
\begin{aligned}
\boldsymbol{J} B & =\left(\boldsymbol{e}_{\varphi} \partial_{\theta}-\boldsymbol{e}_{\theta} \partial_{\varphi}\right) B \\
\nabla \times \boldsymbol{J} A & =\nabla \times(\boldsymbol{x} \times \nabla A)=-\nabla \times(\nabla \times(\boldsymbol{x} A)) \\
& =\Delta(\boldsymbol{x} A)-\nabla(\nabla \cdot(\boldsymbol{x} A)) \\
& =(2 \nabla A+\boldsymbol{x} \Delta A)-\nabla(3 A+\boldsymbol{x} \cdot \nabla A) \\
& =\boldsymbol{x} \Delta A-2 \nabla A-\boldsymbol{x} \cdot \nabla \nabla A=\boldsymbol{x} \Delta A-(2+\boldsymbol{x} \cdot \nabla) \nabla A \\
& =\rho \boldsymbol{\sigma}\left(\left(\partial_{\rho}+\frac{2}{\rho}\right) \partial_{\rho}+\rho^{-2} \Delta_{\sigma}\right) A-\left(2+\rho \partial_{\rho}\right)\left(\boldsymbol{\sigma} \partial_{\rho} A+\rho^{-1} \nabla_{\sigma} A\right) \\
& =\boldsymbol{\sigma} \rho^{-1} \Delta_{\sigma} A-\left(\partial_{\rho}+\frac{1}{\rho}\right) \nabla_{\sigma} A \\
\nabla \times \boldsymbol{J} B & =\boldsymbol{\sigma} \rho^{-1} \Delta_{\sigma} B-\left(\partial_{\rho}+\frac{1}{\rho}\right) \nabla_{\sigma} B \\
\nabla \times(\nabla \times \boldsymbol{J} A) & =\nabla \times(\Delta(\boldsymbol{x} A)-\nabla(\nabla \cdot(\boldsymbol{x} A)))=\nabla \times \Delta(\boldsymbol{x} A) \\
& =\nabla \times(\boldsymbol{x} \Delta A)=-\boldsymbol{x} \times \nabla \Delta A
\end{aligned}
$$

By using these relations and (3.2), we see

$$
\begin{aligned}
& \boldsymbol{x} \cdot \boldsymbol{w}=\boldsymbol{x} \cdot \boldsymbol{u}-\Delta_{\sigma} A=0, \\
& \boldsymbol{x} \cdot(\nabla \times \boldsymbol{w})=\boldsymbol{x} \cdot(\nabla \times \boldsymbol{u})-\Delta_{\sigma} B=0 .
\end{aligned}
$$

Namely, $\boldsymbol{w}$ and $\nabla \times \boldsymbol{w}$ have no radial component. This enables the solenoidal field $\boldsymbol{w}=$ $w_{\theta} \boldsymbol{e}_{\theta}+w_{\varphi} \boldsymbol{e}_{\varphi}$ to satisfy

$$
\left\{\begin{array}{l}
\boldsymbol{x} \cdot(\nabla \times \boldsymbol{w})=D_{\theta} w_{\varphi}-\partial_{\varphi} w_{\theta}=0 \\
\rho \nabla \cdot \boldsymbol{w}=D_{\theta} w_{\theta}+\partial_{\varphi} w_{\varphi}=0
\end{array}\right.
$$

or equivalently

$$
\partial_{\theta}\left(w_{\varphi} \sin \theta\right)-\partial_{\varphi} w_{\theta}=\partial_{\theta}\left(w_{\theta} \sin \theta\right)+\partial_{\varphi} w_{\varphi}=0
$$

This fact implies

$$
\begin{aligned}
\Delta_{\sigma}\left(w_{\theta} \sin \theta\right) & =D_{\theta} \partial_{\theta}\left(w_{\theta} \sin \theta\right)+\partial_{\varphi} \partial_{\varphi} w_{\theta} \\
& =D_{\theta}\left(-\partial_{\varphi} w_{\varphi}\right)+\partial_{\varphi} \partial_{\theta}\left(w_{\varphi} \sin \theta\right)=\left(-D_{\theta} \partial_{\varphi}+\partial_{\varphi} D_{\theta}\right) w_{\varphi}=0, \\
\Delta_{\sigma}\left(w_{\varphi} \sin \theta\right) & =D_{\theta} \partial_{\theta}\left(w_{\varphi} \sin \theta\right)+\partial_{\varphi} \partial_{\varphi} w_{\varphi}=D_{\theta} \partial_{\varphi} w_{\theta}-\partial_{\varphi} \partial_{\theta}\left(w_{\theta} \sin \theta\right) \\
& =\left(D_{\theta} \partial_{\varphi}-\partial_{\varphi} D_{\theta}\right) w_{\theta}=0 .
\end{aligned}
$$

Multiplying by $-w_{\theta} \sin \theta$ or $-w_{\varphi} \sin \theta$, and integrating by parts over $\mathbb{S}^{2}$, we have

$$
\begin{aligned}
& -\int_{\mathbb{S}^{2}}\left(w_{\theta} \sin \theta\right) \Delta_{\sigma}\left(w_{\theta} \sin \theta\right) d \sigma=\int_{\mathbb{S}^{2}}\left|\nabla_{\sigma}\left(w_{\theta} \sin \theta\right)\right|^{2} d \sigma=0, \\
& -\int_{\mathbb{S}^{2}}\left(w_{\varphi} \sin \theta\right) \Delta_{\sigma}\left(w_{\varphi} \sin \theta\right) d \sigma=\int_{\mathbb{S}^{2}}\left|\nabla_{\sigma}\left(w_{\varphi} \sin \theta\right)\right|^{2} d \sigma=0 .
\end{aligned}
$$

Then it turns out that $\nabla_{\sigma}\left(w_{\theta} \sin \theta\right)=\nabla_{\sigma}\left(w_{\varphi} \sin \theta\right)=\mathbf{0}$, that is, $w_{\theta} \sin \theta$ and $w_{\varphi} \sin \theta$ are constant on $\rho \mathbb{S}^{2}$ for each $\rho>0$. (Here $\rho \mathbb{S}^{2}$ denotes the sphere of radius $\rho$ centered at the origin.) Since $w_{\theta}$ and $w_{\varphi}$ are bounded on $\rho \mathbb{S}^{2}$, they does not diverge as $\theta \rightarrow+0$; therefore, such constant numbers must be zero, i.e., $w_{\theta}=w_{\varphi}=0$, which shows that $\boldsymbol{w}=\boldsymbol{e}_{\theta} w_{\theta}+\boldsymbol{e}_{\varphi} w_{\varphi}=\mathbf{0}$. Thus the proof of (3.3) is done.

It remains to show the $L^{2}$-orthogonality of $\boldsymbol{u}_{P}$ and $\boldsymbol{u}_{T}$. To this end, we claim that

$$
\int_{\mathbb{S}^{2}} \nabla_{\sigma} f \cdot \boldsymbol{J} g d \sigma=0 \quad \text { for any } f, g \in C^{\infty}\left(\mathbb{S}^{2}\right) .
$$

Indeed, a calculation of the integrand gives

$$
\begin{aligned}
\nabla_{\sigma} f \cdot \boldsymbol{J} g & =\left(\boldsymbol{e}_{\theta} \partial_{\theta} f+\boldsymbol{e}_{\varphi} \partial_{\varphi} f\right) \cdot\left(\boldsymbol{e}_{\varphi} \partial_{\theta} g-\boldsymbol{e}_{\theta} \partial_{\varphi} g\right) \\
& =-\left(\partial_{\theta} f\right) \partial_{\varphi} g+\left(\partial_{\varphi} f\right) \partial_{\theta} g
\end{aligned}
$$


By integration (by parts) on the both sides over $\mathbb{S}^{2}$, we obtain

$$
\int_{\mathbb{S}^{2}} \nabla_{\sigma} f \cdot \boldsymbol{J} g d \sigma=\int_{\mathbb{S}^{2}} f\left(D_{\theta} \partial_{\varphi}-\partial_{\varphi} \partial_{\theta}\right) g d \sigma=0
$$

which proves the claim. Then we see

$$
\int_{\mathbb{S}^{2}} \boldsymbol{u}_{P} \cdot \boldsymbol{u}_{T} d \sigma=-\int_{\mathbb{S}^{2}}\left(\left(\frac{1}{\rho}+\partial_{\rho}\right) \nabla_{\sigma} A\right) \cdot(\boldsymbol{J} B)=0
$$

by applying the claim for $f=\left(\frac{1}{\rho}+\partial_{\rho}\right) A$ and $g=B$. Likewise, we also have

$$
\int_{\mathbb{S}^{2}} \partial_{\rho} \boldsymbol{u}_{P} \cdot \partial_{\rho} \boldsymbol{u}_{T} d \sigma=-\int_{\mathbb{S}^{2}} \nabla_{\sigma} \partial_{\rho}\left(\left(\frac{1}{\rho}+\partial_{\rho}\right) A\right) \cdot \boldsymbol{J} \partial_{\rho} B d \sigma=0,
$$

and hence

$$
\begin{aligned}
\int_{\mathbb{S}^{2}} \nabla \boldsymbol{u}_{P} \cdot \nabla \boldsymbol{u}_{T} d \sigma & =\int_{\mathbb{S}^{2}} \nabla_{\sigma} \boldsymbol{u}_{P} \cdot \nabla_{\sigma} \boldsymbol{u}_{T} d \sigma=-\int_{\mathbb{S}^{2}} \boldsymbol{u}_{P} \cdot \Delta_{\sigma} \boldsymbol{u}_{T} d \sigma \\
& =-\int_{\mathbb{S}^{2}}\left(\left(\frac{1}{\rho}+\partial_{\rho}\right) \nabla_{\sigma} A\right) \cdot \Delta_{\sigma} \boldsymbol{J} B d \sigma \\
& =-\int_{\mathbb{S}^{2}} \nabla_{\sigma}\left(\left(\frac{1}{\rho}+\partial_{\rho}\right) A\right) \cdot \boldsymbol{J} \Delta_{\sigma} B d \sigma=0 .
\end{aligned}
$$

\section{Proof of Theorem 2: evaluation of the toroidal and Poloidal parts of HARDY-LERAY QUOTIENT}

In the following, we assume $\boldsymbol{u} \not \equiv \mathbf{0}$ and $\int_{\mathbb{R}^{3}}|\nabla \boldsymbol{u}|^{2}|\boldsymbol{x}|^{2 \gamma} d x<\infty$, since otherwise there is nothing to prove. As in [1], there exists an integer $k>-\gamma-\frac{3}{2}$ such that $\nabla \boldsymbol{u}=O\left(|\boldsymbol{x}|^{k}\right)$ as $|\boldsymbol{x}| \rightarrow 0$. It then follows from the condition " $\boldsymbol{u}(\mathbf{0})=\mathbf{0}$ if $\gamma \leq-\frac{1}{2}$ " that

$$
|\boldsymbol{x}|^{\gamma+\frac{1}{2}} \boldsymbol{u}(\boldsymbol{x})=O\left(|\boldsymbol{x}|^{\beta}\right) \quad \text { for } \quad \beta=\left\{\begin{array}{cl}
k+\gamma+\frac{3}{2} & \text { if } \gamma \leq-\frac{1}{2} \\
\gamma+\frac{1}{2} & \text { if } \gamma>-\frac{1}{2}
\end{array}\right.
$$

This ensures, since $\beta>0$, that $\int_{\mathbb{R}^{3}}|\boldsymbol{u}|^{2}|\boldsymbol{x}|^{2 \gamma-2} d x<\infty$. Then it turns out, from the $L^{2}$ orthogonality of the decomposition $\boldsymbol{u}=\boldsymbol{u}_{P}+\boldsymbol{u}_{T}$ in proposition 4 , that

$$
\begin{aligned}
\frac{\int_{\mathbb{R}^{3}}|\nabla \boldsymbol{u}|^{2}|\boldsymbol{x}|^{2 \gamma} d x}{\int_{\mathbb{R}^{3}}|\boldsymbol{u}|^{2}|\boldsymbol{x}|^{2 \gamma-2} d x} & =\frac{\int_{\mathbb{R}^{3}}\left|\nabla \boldsymbol{u}_{P}\right|^{2}|\boldsymbol{x}|^{2 \gamma} d x+\int_{\mathbb{R}^{3}}\left|\nabla \boldsymbol{u}_{T}\right|^{2}|\boldsymbol{x}|^{2 \gamma} d x}{\int_{\mathbb{R}^{3}}\left|\boldsymbol{u}_{P}\right|^{2}|\boldsymbol{x}|^{2 \gamma-2} d x+\int_{\mathbb{R}^{3}}\left|\boldsymbol{u}_{T}\right|^{2}|\boldsymbol{x}|^{2 \gamma-2} d x} \\
& \geq \min \left\{\frac{\int_{\mathbb{R}^{3}}\left|\nabla \boldsymbol{u}_{P}\right|^{2}|\boldsymbol{x}|^{2 \gamma} d x}{\int_{\mathbb{R}^{3}}\left|\boldsymbol{u}_{P}\right|^{2}|\boldsymbol{x}|^{2 \gamma-2} d x}, \frac{\int_{\mathbb{R}^{3}}\left|\nabla \boldsymbol{u}_{T}\right|^{2}|\boldsymbol{x}|^{2 \gamma} d x}{\int_{\mathbb{R}^{3}}\left|\boldsymbol{u}_{T}\right|^{2}|\boldsymbol{x}|^{2 \gamma-2} d x}\right\}
\end{aligned}
$$

if $\boldsymbol{u}_{T} \not \equiv \mathbf{0}$ and $\boldsymbol{u}_{P} \not \equiv \mathbf{0}$. Namely, the Hardy-Leray quotient for a solenoidal field is estimated from bellow by the lesser of that for poloidal part and for toroidal part. Now, all we have to do is to evaluate them separately.

4.1. Evaluation of the toroidal part. Let $\boldsymbol{u}_{T}=\boldsymbol{J} B \not \equiv \mathbf{0}$. By using the formula

$$
\boldsymbol{J} f \cdot \boldsymbol{J} g=\nabla_{\sigma} f \cdot \nabla_{\sigma} g \quad \text { for any } f, g \in C^{\infty}\left(\mathbb{S}^{2}\right)
$$


we write the $L^{2}\left(\mathbb{S}^{2}\right)$-integrals of $\boldsymbol{u}_{T}$ and $\rho \nabla \boldsymbol{u}_{T}$ in terms of the toroidal potential $B$ as

$$
\begin{aligned}
\int_{\mathbb{S}^{2}}\left|\boldsymbol{u}_{T}\right|^{2} d \sigma= & \int_{\mathbb{S}^{2}}|\boldsymbol{J} B|^{2} d \sigma=\int_{\mathbb{S}^{2}}\left|\nabla_{\sigma} B\right|^{2} d \sigma \\
\rho^{2} \int_{\mathbb{S}^{2}}\left|\nabla \boldsymbol{u}_{T}\right|^{2} d \sigma & =\int_{\mathbb{S}^{2}}\left(\left|\rho \partial_{\rho} \boldsymbol{u}_{T}\right|^{2}+\left|\nabla_{\sigma} \boldsymbol{u}_{T}\right|^{2}\right) d \sigma=\int_{\mathbb{S}^{2}}\left(\left|\rho \partial_{\rho} \boldsymbol{J} B\right|^{2}+\left|\nabla_{\sigma} \boldsymbol{J} B\right|^{2}\right) d \sigma \\
& =\int_{\mathbb{S}^{2}}\left(\rho^{2}\left|\boldsymbol{J} \partial_{\rho} B\right|^{2}-\boldsymbol{J} B \cdot \boldsymbol{J} \Delta_{\sigma} B\right) d \sigma \\
& =\int_{\mathbb{S}^{2}}\left(\rho^{2}\left|\nabla_{\sigma} \partial_{\rho} B\right|^{2}-\nabla_{\sigma} B \cdot \nabla_{\sigma} \Delta_{\sigma} B\right) d \sigma \\
& =\int_{\mathbb{S}^{2}}\left(\left(\Delta_{\sigma} B\right)^{2}+\rho^{2}\left|\partial_{\rho} \nabla_{\sigma} B\right|^{2}\right) d \sigma .
\end{aligned}
$$

Now let us define the function $f \in C^{\infty}\left(\mathbb{R}^{3} \backslash\{\mathbf{0}\}\right)$ by

$$
f(\boldsymbol{x})=\rho^{\gamma+\frac{1}{2}} B=|\boldsymbol{x}|^{\gamma+\frac{1}{2}} G(\nabla \times \boldsymbol{u})(\boldsymbol{x}),
$$

which is also continuous with compact support on $\mathbb{R}^{3}$; we note, for $\beta>0$ given in (4.1), that

$$
\left.\begin{array}{rl}
f(\boldsymbol{x}) & =O\left(|\boldsymbol{x}|^{\beta}\right), \\
|\boldsymbol{x}| \nabla f(\boldsymbol{x}) & =O\left(|\boldsymbol{x}|^{\beta}\right)
\end{array}\right\} \quad \text { as }|\boldsymbol{x}| \rightarrow+0,
$$

which we can verify by using (3.1) with $\boldsymbol{u}$ replaced by $\nabla \times \boldsymbol{u}$. Integrating the above $L^{2}\left(\mathbb{S}^{2}\right)$ equations with measure $\rho^{2 \gamma} d \rho$ over $\mathbb{R}_{+}$and transforming $B$ into $f$, we see

$$
\begin{aligned}
\int_{\mathbb{R}^{3}}\left|\boldsymbol{u}_{T}\right|^{2}|\boldsymbol{x}|^{2 \gamma-2} d x & =\int_{0}^{\infty} \rho^{2 \gamma} d \rho \int_{\mathbb{S}^{2}}\left|\nabla_{\sigma} B\right|^{2} d \sigma=\int_{0}^{\infty} \frac{d \rho}{\rho} \int_{\mathbb{S}^{2}}\left|\nabla_{\sigma} f\right|^{2} d \sigma, \\
\int_{\mathbb{R}^{3}}\left|\nabla \boldsymbol{u}_{T}\right|^{2}|\boldsymbol{x}|^{2 \gamma} d x & =\int_{0}^{\infty} \frac{d \rho}{\rho} \int_{\mathbb{S}^{2}}\left(\Delta_{\sigma} f\right)^{2} d \sigma+\int_{\mathbb{S}^{2}} d \sigma \int_{0}^{\infty} \rho^{2 \gamma+2}\left|\partial_{\rho} \nabla_{\sigma} B\right|^{2} d \rho .
\end{aligned}
$$

Here the last radial integration is written in terms of $f$ as

$$
\begin{aligned}
\int_{0}^{\infty} \rho^{2 \gamma+2}\left|\partial_{\rho} \nabla_{\sigma} B\right|^{2} d \rho & =\int_{0}^{\infty}\left|\rho \partial_{\rho} \nabla_{\sigma}\left(\rho^{-\gamma-\frac{1}{2}} f\right)\right|^{2} \rho^{2 \gamma} d \rho \\
& =\int_{0}^{\infty}\left|\left(-\gamma-\frac{1}{2}\right) \nabla_{\sigma} f+\rho \partial_{\rho} \nabla_{\sigma} f\right|^{2} \frac{d \rho}{\rho} \\
& =\int_{0}^{\infty}\left(\left(\gamma+\frac{1}{2}\right)^{2}\left|\nabla_{\sigma} f\right|^{2}+\left|\rho \partial_{\rho} \nabla_{\sigma} f\right|^{2}\right) \frac{d \rho}{\rho}-\left(\gamma+\frac{1}{2}\right) \int_{0}^{\infty} \partial_{\rho}\left|\nabla_{\sigma} f\right|^{2} d \rho \\
& =\int_{0}^{\infty}\left(\left(\gamma+\frac{1}{2}\right)^{2}\left|\nabla_{\sigma} f\right|^{2}+\left|\rho \partial_{\rho} \nabla_{\sigma} f\right|^{2}\right) \frac{d \rho}{\rho} \quad(\text { by use of }(4.3)) .
\end{aligned}
$$

It then follows, by combining the above integral equalities, that

$$
\begin{aligned}
\frac{\int_{\mathbb{R}^{3}}\left|\nabla \boldsymbol{u}_{T}\right|^{2}|\boldsymbol{x}|^{2 \gamma} d x}{\int_{\mathbb{R}^{3}}\left|\boldsymbol{u}_{T}\right|^{2}|\boldsymbol{x}|^{2 \gamma-2} d x} & =\frac{\int_{0}^{\infty} \frac{d \rho}{\rho} \int_{\mathbb{S}^{2}}\left(\Delta_{\sigma} f\right)^{2} d \sigma+\int_{\mathbb{S}^{2}} d \sigma \int_{0}^{\infty}\left(\left(\gamma+\frac{1}{2}\right)^{2}\left|\nabla_{\sigma} f\right|^{2}+\left|\rho \partial_{\rho} \nabla_{\sigma} f\right|^{2}\right) \frac{d \rho}{\rho}}{\int_{0}^{\infty} \frac{d \rho}{\rho} \int_{\mathbb{S}^{2}}\left|\nabla_{\sigma} f\right|^{2} d \sigma} \\
& \geq\left(\gamma+\frac{1}{2}\right)^{2}+\frac{\int_{0}^{\infty} \frac{d \rho}{\rho} \int_{\mathbb{S}^{2}}\left(\Delta_{\sigma} f\right)^{2} d \sigma}{\int_{0}^{\infty} \frac{d \rho}{\rho} \int_{\mathbb{S}^{2}}\left|\nabla_{\sigma} f\right|^{2} d \sigma}
\end{aligned}
$$

In order to evaluate the last term, we use the spherical harmonics expansion of $f$ :

$$
f(\rho \boldsymbol{\sigma})=\sum_{\nu=0}^{\infty} \sum_{m=-\nu}^{\nu} f_{\nu m}(\rho) Y_{\nu m}(\theta, \varphi), \quad\left\{\begin{array}{c}
-\Delta_{\sigma} Y_{\nu m}=\alpha_{\nu} Y_{\nu m}, \\
J_{1} Y_{\nu m}=i m Y_{\nu m} \\
\alpha_{\nu}=\nu(\nu+1) .
\end{array}\right.
$$


Then we have

$$
\begin{aligned}
\frac{\int_{0}^{\infty} \frac{d \rho}{\rho} \int_{\mathbb{S}^{2}}\left(\Delta_{\sigma} f\right)^{2} d \sigma}{\int_{0}^{\infty} \frac{d \rho}{\rho} \int_{\mathbb{S}^{2}}\left|\nabla_{\sigma} f\right|^{2} d \sigma} & =\frac{\int_{0}^{\infty} \frac{d \rho}{\rho} \int_{\mathbb{S}^{2}} f\left(-\Delta_{\sigma}\right)^{2} f d \sigma}{\int_{0}^{\infty} \frac{d \rho}{\rho} \int_{\mathbb{S}^{2}} f\left(-\Delta_{\sigma}\right) f d \sigma}=\frac{\sum_{\nu=0}^{\infty} \sum_{m=-\nu}^{\nu} \alpha_{\nu}^{2} \int_{0}^{\infty}\left|f_{\nu m}(\rho)\right|^{2} \frac{d \rho}{\rho}}{\sum_{\nu=0}^{\infty} \sum_{m=-\nu}^{\nu} \alpha_{\nu} \int_{0}^{\infty}\left|f_{\nu m}(\rho)\right|^{2} \frac{d \rho}{\rho}} \\
& =\frac{\sum_{\nu=1}^{\infty} \sum_{m=-\nu}^{\nu} \alpha_{\nu}^{2} \int_{0}^{\infty}\left|f_{\nu m}(\rho)\right|^{2} \frac{d \rho}{\rho}}{\sum_{\nu=1}^{\infty} \sum_{m=-\nu}^{\nu} \alpha_{\nu} \int_{0}^{\infty}\left|f_{\nu m}(\rho)\right|^{2} \frac{d \rho}{\rho}} \geq \inf _{\nu \in \mathbb{N}} \frac{\alpha_{\nu}^{2}}{\alpha_{\nu}}=\alpha_{1}
\end{aligned}
$$

Hence the toroidal part of the Hardy-Leray quotient is estimated by

$$
\frac{\int_{\mathbb{R}^{3}}\left|\nabla \boldsymbol{u}_{T}\right|^{2}|\boldsymbol{x}|^{2 \gamma} d x}{\int_{\mathbb{R}^{3}}\left|\boldsymbol{u}_{T}\right|^{2}|\boldsymbol{x}|^{2 \gamma-2} d x} \geq\left(\gamma+\frac{1}{2}\right)^{2}+\alpha_{1} .
$$

4.2. Evaluation of the poloidal part. Let $\boldsymbol{u}_{P}=\nabla \times \boldsymbol{J} A \not \equiv \mathbf{0}$. Let us transform the potential $A$ into the function $f \in C^{\infty}\left(\mathbb{R}^{3} \backslash\{\mathbf{0}\}\right)$ by the formula

$$
f(\boldsymbol{x})=\rho^{\gamma-\frac{1}{2}} A=|\boldsymbol{x}|^{\gamma-\frac{1}{2}} G \boldsymbol{u}(\boldsymbol{x}),
$$

which is also continuous with compact support on $\mathbb{R}^{3}$; we note, for $\beta>0$ in (4.1), that

$$
\left.\begin{array}{r}
f(\boldsymbol{x})=O\left(|\boldsymbol{x}|^{\beta}\right), \\
|\boldsymbol{x}| \nabla f(\boldsymbol{x})=O\left(|\boldsymbol{x}|^{\beta}\right), \\
|\boldsymbol{x}|^{2} \nabla^{2} f(\boldsymbol{x})=O\left(|\boldsymbol{x}|^{\beta}\right)
\end{array}\right\} \quad \text { as }|\boldsymbol{x}| \rightarrow+0,
$$

which can be verified by using (3.1). Then the poloidal part of $\boldsymbol{u}$ is expressed in terms of $f$ as

$$
\begin{aligned}
\boldsymbol{u}_{P} & =\left(\frac{\boldsymbol{\sigma}}{\rho} \Delta_{\sigma}-\left(\frac{1}{\rho}+\partial_{\rho}\right) \nabla_{\sigma}\right)\left(\rho^{\frac{1}{2}-\gamma} f\right) \\
& =\rho^{-\frac{1}{2}-\gamma}\left(\boldsymbol{\sigma} \Delta_{\sigma} f-\left(\frac{3}{2}-\gamma+\rho \partial_{\rho}\right) \nabla_{\sigma} f\right) \\
& =\rho^{-\frac{1}{2}-\gamma}\left(\boldsymbol{\sigma} \Delta_{\sigma} f-\left(\frac{3}{2}-\gamma+\partial_{t}\right) \nabla_{\sigma} f\right)
\end{aligned}
$$

where we have introduced the deformed radial coordinate $t \in \mathbb{R}$ by the transformation

$$
t=\log \rho,
$$

whose differential obeys the chain rules: $\rho \partial_{\rho}=\partial_{t}, d t=\frac{d \rho}{\rho}$; hereafter we prefer to use $t$ as an alternative radial coordinate, rather than $\rho$.

Taking the absolute square of (4.7) yields

$$
\rho^{2 \gamma+1}\left|\boldsymbol{u}_{P}\right|^{2}=\left(\Delta_{\sigma} f\right)^{2}+\left(\frac{3}{2}-\gamma\right)^{2}\left|\nabla_{\sigma} f\right|^{2}+\left|\partial_{t} \nabla_{\sigma} f\right|^{2}-\left(\frac{3}{2}-\gamma\right) \frac{\partial}{\partial t}\left|\nabla_{\sigma} f\right|^{2}
$$

Integrating the both sides with measure $|\boldsymbol{x}|^{-3} d x=\frac{d \rho}{\rho} d \sigma=d t d \sigma$, we can express the $L^{2}\left(|\boldsymbol{x}|^{2 \gamma} d x\right)$ integral of $\boldsymbol{u}_{P} /|\boldsymbol{x}|$ as

$$
\begin{aligned}
\int_{\mathbb{R}^{3}} \frac{\left|\boldsymbol{u}_{P}\right|^{2}}{|\boldsymbol{x}|^{2}}|\boldsymbol{x}|^{2 \gamma} d x & =\iint_{\mathbb{R}_{+} \times \mathbb{S}^{2}} \rho^{2 \gamma+1}\left|\boldsymbol{u}_{P}\right|^{2} \frac{d \rho}{\rho} d \sigma=\iint_{\mathbb{R} \times \mathbb{S}^{2}} \rho^{2 \gamma+1}\left|\boldsymbol{u}_{P}\right|^{2} d t d \sigma \\
& =\int_{\mathbb{R}} d t \int_{\mathbb{S}^{2}}\left(\left(\Delta_{\sigma} f\right)^{2}+\left(\frac{3}{2}-\gamma\right)^{2}\left|\nabla_{\sigma} f\right|^{2}+\left|\partial_{t} \nabla_{\sigma} f\right|^{2}\right) d \sigma
\end{aligned}
$$

by use of (4.6).

In order to calculate the $L^{2}\left(|\boldsymbol{x}|^{2 \gamma} d x\right)$-integral of $\nabla \boldsymbol{u}_{P}$, we start with calculation of its spherical part; we see, by using (2.7) and (4.7), that

$$
\begin{aligned}
\Delta_{\sigma}\left(\rho^{\gamma+\frac{1}{2}} \boldsymbol{u}_{P}\right) & =\Delta_{\sigma}\left(\boldsymbol{\sigma} \Delta_{\sigma} f\right)-\left(\frac{3}{2}-\gamma+\partial_{t}\right) \Delta_{\sigma} \nabla_{\sigma} f \\
& =\boldsymbol{\sigma} \Delta_{\sigma}^{2} f+2\left(\nabla_{\sigma}-\boldsymbol{\sigma}\right) \Delta_{\sigma} f-\left(\frac{3}{2}-\gamma+\partial_{t}\right)\left(\nabla_{\sigma} \Delta_{\sigma} f-2 \boldsymbol{\sigma} \Delta_{\sigma} f\right) \\
& =\boldsymbol{\sigma}\left(\Delta_{\sigma}^{2} f+2\left(\frac{1}{2}-\gamma+\partial_{t}\right) \Delta_{\sigma} f\right)+\left(\frac{1}{2}+\gamma-\partial_{t}\right) \nabla_{\sigma} \Delta_{\sigma} f .
\end{aligned}
$$


Multiplication by $-\rho^{\gamma+\frac{1}{2}} \boldsymbol{u}_{P} \cdot$ and integration by parts over $\mathbb{S}^{2}$ yield

$$
\begin{aligned}
\int_{\mathbb{S}^{2}} & \left|\nabla_{\sigma} \boldsymbol{u}_{P}\right|^{2} \rho^{2 \gamma+1} d \sigma=-\int_{\mathbb{S}^{2}} \rho^{\gamma+\frac{1}{2}} \boldsymbol{u}_{P} \cdot \Delta_{\sigma}\left(\rho^{\gamma+\frac{1}{2}} \boldsymbol{u}_{P}\right) d \sigma \\
= & -\int_{\mathbb{S}^{2}}\left(\left(\Delta_{\sigma} f\right)\left(\Delta_{\sigma}^{2} f+2\left(\frac{1}{2}-\gamma+\partial_{t}\right) \Delta_{\sigma} f\right)-\left(\frac{3}{2}-\gamma+\partial_{t}\right) \nabla_{\sigma} f \cdot\left(\frac{1}{2}+\gamma-\partial_{t}\right) \nabla_{\sigma} \Delta_{\sigma} f\right) d \sigma \\
= & -\int_{\mathbb{S}^{2}}\left(\left(\Delta_{\sigma} f\right) \Delta_{\sigma}^{2} f+2\left(\frac{1}{2}-\gamma\right)\left(\Delta_{\sigma} f\right)^{2}+\left(\frac{3}{2}-\gamma\right)\left(\frac{1}{2}+\gamma\right)\left(\Delta_{\sigma} f\right)^{2}-\left(\partial_{t} \Delta_{\sigma} f\right)^{2}\right) d \sigma \\
& -\int_{\mathbb{S}^{2}}\left(\left(\Delta_{\sigma} f\right) 2 \partial_{t} \Delta_{\sigma} f+\left(\frac{3}{2}-\gamma\right)\left(\nabla_{\sigma} f\right) \cdot \partial_{t} \nabla_{\sigma} \Delta_{\sigma} f-\left(\partial_{t} \nabla_{\sigma} f\right) \cdot\left(\frac{1}{2}+\gamma\right) \nabla_{\sigma} \Delta_{\sigma} f\right) d \sigma \\
= & \int_{\mathbb{S}^{2}}\left(\left|\nabla_{\sigma} \Delta_{\sigma} f\right|^{2}+\left(\left(\gamma+\frac{1}{2}\right)^{2}-2\right)\left(\Delta_{\sigma} f\right)^{2}+\left(\partial_{t} \Delta_{\sigma} f\right)^{2}\right) d \sigma-\left(\gamma+\frac{1}{2}\right) \frac{d}{d t} \int_{\mathbb{S}^{2}}\left(\Delta_{\sigma} f\right)^{2} d \sigma .
\end{aligned}
$$

Integrating this with measure $\frac{d \rho}{\rho}=d t$ over $\mathbb{R}_{+} \cong \mathbb{R}$, we obtain

$$
\begin{gathered}
\int_{\mathbb{R}^{3}}\left|\nabla_{\sigma} \boldsymbol{u}_{P}\right|^{2}|\boldsymbol{x}|^{2 \gamma-2} d x=\int_{0}^{\infty} \frac{d \rho}{\rho} \int_{\mathbb{S}^{2}}\left|\nabla_{\sigma} \boldsymbol{u}_{P}\right|^{2} \rho^{2 \gamma+1} d \sigma=\int_{\mathbb{R}} d t \int_{\mathbb{S}^{2}}\left|\nabla_{\sigma} \boldsymbol{u}_{P}\right|^{2} \rho^{2 \gamma+1} d \sigma \\
=\iint_{\mathbb{R} \times \mathbb{S}^{2}}\left(\left|\nabla_{\sigma} \Delta_{\sigma} f\right|^{2}+\left(\left(\gamma+\frac{1}{2}\right)^{2}-2\right)\left(\Delta_{\sigma} f\right)^{2}+\left(\partial_{t} \Delta_{\sigma} f\right)^{2}\right) d t d \sigma
\end{gathered}
$$

by use of (4.6). Also, we similarly calculate the radial part of $\nabla \boldsymbol{u}_{P}$; we see, by (4.7), that

$$
\begin{aligned}
\rho^{\gamma+\frac{1}{2}} \rho \partial_{\rho} \boldsymbol{u}_{P} & =\rho^{\gamma+\frac{1}{2}} \rho \partial_{\rho}\left(\rho^{-\frac{1}{2}-\gamma}\left(\boldsymbol{\sigma} \Delta_{\sigma}-\left(\frac{3}{2}-\gamma+\partial_{t}\right) \nabla_{\sigma}\right) f\right) \\
& =\left(-\frac{1}{2}-\gamma\right)\left(\boldsymbol{\sigma} \Delta_{\sigma}-\left(\frac{3}{2}-\gamma+\partial_{t}\right) \nabla_{\sigma}\right) f+\left(\boldsymbol{\sigma} \partial_{t} \Delta_{\sigma}-\left(\frac{3}{2}-\gamma+\partial_{t}\right) \partial_{t} \nabla_{\sigma}\right) f \\
& =\boldsymbol{\sigma}\left(-\frac{1}{2}-\gamma+\partial_{t}\right) \Delta_{\sigma} f+\left(\left(\frac{1}{2}+\gamma\right)\left(\frac{3}{2}-\gamma\right)+(2 \gamma-1) \partial_{t}-\partial_{t}^{2}\right) \nabla_{\sigma} f .
\end{aligned}
$$

Taking the absolute square of this yields

$$
\begin{aligned}
\left|\rho^{\frac{1}{2}+\gamma} \rho \partial_{\rho} \boldsymbol{u}_{P}\right|^{2}= & \left(\left(-\frac{1}{2}-\gamma+\partial_{t}\right) \Delta_{\sigma} f\right)^{2}+\left|\left(\left(\frac{1}{2}+\gamma\right)\left(\frac{3}{2}-\gamma\right)+(2 \gamma-1) \partial_{t}-\partial_{t}^{2}\right) \nabla_{\sigma} f\right|^{2} \\
= & \left(\gamma+\frac{1}{2}\right)^{2}\left(\left(\Delta_{\sigma} f\right)^{2}+\left(\frac{3}{2}-\gamma\right)^{2}\left|\nabla_{\sigma} f\right|^{2}\right)+\left(\partial_{t} \Delta_{\sigma} f\right)^{2} \\
& +\left((2 \gamma-1)^{2}+2\left(\frac{1}{2}+\gamma\right)\left(\frac{3}{2}-\gamma\right)\right)\left|\partial_{t} \nabla_{\sigma} f\right|^{2}+\left|\partial_{t}^{2} \nabla_{\sigma} f\right|^{2} \\
& +\frac{\partial}{\partial t}\left(\left(-\frac{1}{2}-\gamma\right)\left(\Delta_{\sigma} f\right)^{2}+\left(\frac{1}{2}+\gamma\right)\left(\frac{3}{2}-\gamma\right)(2 \gamma-1)\left|\nabla_{\sigma} f\right|^{2}\right. \\
& \left.\quad-(2 \gamma-1)\left|\partial_{t} \nabla_{\sigma} f\right|^{2}-2\left(\frac{1}{2}+\gamma\right)\left(\frac{3}{2}-\gamma\right) \nabla_{\sigma} f \cdot \partial_{t} \nabla_{\sigma} f\right) .
\end{aligned}
$$

By integration of this with measure $\frac{d \rho}{\rho}=d t$ over $\mathbb{R}_{+} \cong \mathbb{R}$, and by use of (4.6), we obtain

$$
\begin{aligned}
& \int_{\mathbb{R}_{+}}\left|\partial_{\rho} \boldsymbol{u}_{P}\right|^{2} \rho^{2 \gamma} \rho^{2} d \rho=\int_{\mathbb{R}}((\gamma\left.+\frac{1}{2}\right)^{2}\left(\left(\Delta_{\sigma} f\right)^{2}+\left(\frac{3}{2}-\gamma\right)^{2}\left|\nabla_{\sigma} f\right|^{2}\right)+\left(\partial_{t} \Delta_{\sigma} f\right)^{2} \\
&\left.+\left((2 \gamma-1)^{2}+2\left(\frac{1}{2}+\gamma\right)\left(\frac{3}{2}-\gamma\right)\right)\left|\partial_{t} \nabla_{\sigma} f\right|^{2}+\left|\partial_{t}^{2} \nabla_{\sigma} f\right|^{2}\right) d t \\
&=\int_{\mathbb{R}}\left(\left(\gamma+\frac{1}{2}\right)^{2}\left(\left(\Delta_{\sigma} f\right)^{2}+\left(\frac{3}{2}-\gamma\right)^{2}\left|\nabla_{\sigma} f\right|^{2}+\left|\partial_{t} \nabla_{\sigma} f\right|^{2}\right)\right.\left.+\left(\frac{3}{2}-\gamma\right)^{2}\left|\partial_{t} \nabla_{\sigma} f\right|^{2}+\left(\partial_{t} \Delta_{\sigma} f\right)^{2}+\left|\partial_{t}^{2} \nabla_{\sigma} f\right|^{2}\right) d t \\
&=\left(\gamma+\frac{1}{2}\right)^{2} \int_{\mathbb{R}_{+}}\left|\boldsymbol{u}_{P}\right|^{2} \rho^{2 \gamma} d \rho+\int_{\mathbb{R}}\left(\left(\partial_{t} \Delta_{\sigma} f\right)^{2}+\left(\frac{3}{2}-\gamma\right)^{2}\left|\partial_{t} \nabla_{\sigma} f\right|^{2}+\left|\partial_{t}^{2} \nabla_{\sigma} f\right|^{2}\right) d t
\end{aligned}
$$

Therefore, with the abbreviation

$$
\text { r.h.s. }(\cdots):=\text { right-hand side of }(\cdots) \text {, }
$$


the Hardy-Leray quotient for $\boldsymbol{u}_{P} \not \equiv \mathbf{0}$ is expressed as

$$
\begin{aligned}
& \frac{\int_{\mathbb{R}^{3}}\left|\nabla \boldsymbol{u}_{P}\right|^{2}|\boldsymbol{x}|^{2 \gamma} d x}{\int_{\mathbb{R}^{3}}\left|\boldsymbol{u}_{P}\right|^{2}|\boldsymbol{x}|^{2 \gamma-2} d x}=\frac{\text { r.h.s.(4.9) }+\int_{\mathbb{S}^{2}} \text { r.h.s.(4.10)d }}{\text { r.h.s.(4.8) }}=\left(\gamma+\frac{1}{2}\right)^{2}+R_{\gamma}(f) \text {, } \\
& R_{\gamma}(f):=\frac{\iint_{\mathbb{R} \times \mathbb{S}^{2}}\left(\left|\nabla_{\sigma} \Delta_{\sigma} f\right|^{2}+\left(\left(\gamma+\frac{1}{2}\right)^{2}-2\right)\left(\Delta_{\sigma} f\right)^{2}+2\left(\partial_{t} \Delta_{\sigma} f\right)^{2}+\left(\frac{3}{2}-\gamma\right)^{2}\left|\partial_{t} \nabla_{\sigma} f\right|^{2}+\left|\partial_{t}^{2} \nabla_{\sigma} f\right|^{2}\right) d t d \sigma}{\iint_{\mathbb{R} \times \mathbb{S}^{2}}\left(\left(\Delta_{\sigma} f\right)^{2}+\left(\frac{3}{2}-\gamma\right)^{2}\left|\nabla_{\sigma} f\right|^{2}+\left|\partial_{t} \nabla_{\sigma} f\right|^{2}\right) d t d \sigma} .
\end{aligned}
$$

In order to evaluate $R_{\gamma}(f)$, we take the one-dimensional Fourier transformation

$$
f(\boldsymbol{x})=f\left(e^{t} \boldsymbol{\sigma}\right) \mapsto \widehat{f}(\lambda, \boldsymbol{\sigma})=\frac{1}{\sqrt{2 \pi}} \int_{\mathbb{R}} e^{-i \lambda t} f\left(e^{t} \boldsymbol{\sigma}\right) d t .
$$

This operation commutes with the spherical derivative operator: $\widehat{\nabla_{\sigma} f}=\nabla_{\sigma} \widehat{f}$. Also, the radial $t$-derivative is changed into the algebraic multiplier: $\widehat{\partial_{t} f}=i \lambda \widehat{f}$. By the $L^{2}$-isometry of the Fourier transformation, we can then rewrite $R_{\gamma}(f)$ as

$$
R_{\gamma}(f)=\frac{\iint_{\mathbb{R} \times \mathbb{S}^{2}}\left(\left|\nabla_{\sigma} \Delta_{\sigma} \widehat{f}\right|^{2}+\left(\left(\gamma+\frac{1}{2}\right)^{2}-2+2 \lambda^{2}\right)\left|\Delta_{\sigma} \widehat{f}\right|^{2}+\left(\left(\frac{3}{2}-\gamma\right)^{2} \lambda^{2}+\lambda^{4}\right)\left|\nabla_{\sigma} \widehat{f}\right|^{2}\right) d \lambda d \sigma}{\iint_{\mathbb{R} \times \mathbb{S}^{2}}\left(\left|\Delta_{\sigma} \widehat{f}\right|^{2}+\left(\left(\frac{3}{2}-\gamma\right)^{2}+\lambda^{2}\right)\left|\nabla_{\sigma} \widehat{f}\right|^{2}\right) d \lambda d \sigma} .
$$

The $\mathbb{S}^{2}$ integration by parts in each of the denominator and the numerator leads to

$$
R_{\gamma}(f)=\frac{\iint_{\mathbb{R} \times \mathbb{S}^{2}} \overline{\hat{f}} Q\left(\lambda,-\Delta_{\sigma}\right) \widehat{f} d \lambda d \sigma}{\iint_{\mathbb{R} \times \mathbb{S}^{2}} \widehat{\widehat{f}} q\left(\lambda,-\Delta_{\sigma}\right) \widehat{f} d \lambda d \sigma},
$$

where $Q$ and $q$ are the polynomials defined by

$$
\left\{\begin{array}{l}
Q(\lambda, \alpha)=\alpha^{3}+\left(\left(\gamma+\frac{1}{2}\right)^{2}-2+2 \lambda^{2}\right) \alpha^{2}+\left(\left(\frac{3}{2}-\gamma\right)^{2} \lambda^{2}+\lambda^{4}\right) \alpha \\
q(\lambda, \alpha)=\alpha^{2}+\left(\left(\frac{3}{2}-\gamma\right)^{2}+\lambda^{2}\right) \alpha
\end{array}\right.
$$

We now expand $\widehat{f}$ in the spherical harmonics $\left\{Y_{\nu m}\right\}$ as in (4.4). Then we have

$$
\begin{aligned}
\widehat{f}(\lambda, \boldsymbol{\sigma}) & =\sum_{\nu=0}^{\infty} \sum_{m=-\nu}^{\nu} \widehat{f}_{\nu m}(\lambda) Y_{\nu m}(\theta, \varphi), \\
R_{\gamma}(f) & =\frac{\sum_{\nu=0}^{\infty} \sum_{m=-\nu}^{\nu} \int_{\mathbb{R}} Q\left(\lambda, \alpha_{\nu}\right)\left|\widehat{f}_{\nu m}(\lambda)\right|^{2} d \lambda}{\sum_{\nu=0}^{\infty} \sum_{m=-\nu}^{\nu} \int_{\mathbb{R}} q\left(\lambda, \alpha_{\nu}\right)\left|\widehat{f}_{\nu m}(\lambda)\right|^{2} d \lambda} \\
& =\frac{\sum_{\nu=1}^{\infty} \sum_{m=-\nu}^{\nu} \int_{\mathbb{R}} Q\left(\lambda, \alpha_{\nu}\right)\left|\widehat{f}_{\nu m}(\lambda)\right|^{2} d \lambda}{\sum_{\nu=1}^{\infty} \sum_{m=-\nu}^{\nu} \int_{\mathbb{R}} q\left(\lambda, \alpha_{\nu}\right)\left|\widehat{f}_{\nu m}(\lambda)\right|^{2} d \lambda} \\
& \geq \inf _{\lambda \in \mathbb{R}} \inf _{\nu \in \mathbb{N}} \frac{Q\left(\lambda, \alpha_{\nu}\right)}{q\left(\lambda, \alpha_{\nu}\right)}=\inf _{x \geq 0} \inf _{\nu \in \mathbb{N}} F_{\gamma}\left(x, \alpha_{\nu}\right),
\end{aligned}
$$

where

$$
\begin{aligned}
F_{\gamma}\left(x, \alpha_{\nu}\right) & :=\frac{Q\left(\sqrt{x}, \alpha_{\nu}\right)}{q\left(\sqrt{x}, \alpha_{\nu}\right)}=\frac{\alpha_{\nu}^{3}+\left(\left(\gamma+\frac{1}{2}\right)^{2}-2+2 x\right) \alpha_{\nu}^{2}+\left(\left(\frac{3}{2}-\gamma\right)^{2} x+x^{2}\right) \alpha_{\nu}}{\alpha_{\nu}^{2}+\left(\left(\frac{3}{2}-\gamma\right)^{2}+x\right) \alpha_{\nu}} \\
& =x+\alpha_{\nu}\left(1-\frac{4(1-\gamma)}{x+\alpha_{\nu}+\left(\frac{3}{2}-\gamma\right)^{2}}\right),
\end{aligned}
$$

which is just the same as [1, Eq. $\left.(2.36)_{n=3}\right]$. As in [1] again, by checking that

$$
\begin{cases}F_{\gamma}\left(x, \alpha_{\nu}\right) \geq F_{\gamma}\left(0, \alpha_{\nu}\right) \geq F_{\gamma}\left(0, \alpha_{1}\right), & \text { for } \gamma \leq 1, \\ \frac{\partial F_{\gamma}\left(x, \alpha_{\nu}\right)}{\partial \alpha_{\nu}}=1+\frac{4(\gamma-1)\left(x+\left(\frac{3}{2}-\gamma\right)^{2}\right)}{\left(x+\alpha_{\nu}+\left(\frac{3}{2}-\gamma\right)^{2}\right)^{2}}>0, & \text { for } \gamma>1,\end{cases}
$$


we obtain the minimum value of $F_{\gamma}\left(x, \alpha_{\nu}\right)$ :

$$
\begin{aligned}
\inf _{x \geq 0} \inf _{\nu \in \mathbb{N}} F_{\gamma}\left(x, \alpha_{\nu}\right) & =\min _{x \geq 0} F_{\gamma}\left(x, \alpha_{1}\right) \\
& = \begin{cases}F_{\gamma}\left(0, \alpha_{1}\right)=\alpha_{1}\left(1-\frac{4(1-\gamma)}{\alpha_{1}+\left(\frac{3}{2}-\gamma\right)^{2}}\right), & \text { for } \gamma \leq 1 \\
\alpha_{1}+\min _{x \geq 0}\left(x+\frac{4(\gamma-1) \alpha_{1}}{x+\alpha_{1}+\left(\frac{3}{2}-\gamma\right)^{2}}\right), & \text { for } \gamma>1 .\end{cases}
\end{aligned}
$$

Then it follows, by combining (4.12) with (4.11), that the poloidal part of Hardy-Leray quotient is estimated by

$$
\frac{\int_{\mathbb{R}^{3}}\left|\nabla \boldsymbol{u}_{P}\right|^{2}|\boldsymbol{x}|^{2 \gamma} d x}{\int_{\mathbb{R}^{3}}\left|\boldsymbol{u}_{P}\right|^{2}|\boldsymbol{x}|^{2 \gamma-2} d x} \geq\left(\gamma+\frac{1}{2}\right)^{2}+\min _{x \geq 0} F_{\gamma}\left(x, \alpha_{1}\right)
$$

where the value of $\min _{x \geq 0} F_{\gamma}\left(0, \alpha_{1}\right)$ is given in the right-hand side of (4.13).

4.3. Conclusion. By combining (4.5),(4.14) with (4.2), we arrive at

$$
\begin{aligned}
\frac{\int_{\mathbb{R}^{3}}|\nabla \boldsymbol{u}|^{2}|\boldsymbol{x}|^{2 \gamma} d x}{\int_{\mathbb{R}^{3}}|\boldsymbol{u}|^{2}|\boldsymbol{x}|^{2 \gamma-2} d x} \geq & \min \{\text { r.h.s.(4.5), r.h.s. }(4.14)\}=\left(\gamma+\frac{1}{2}\right)^{2}+\min \left\{\alpha_{1}, \min _{x \geq 0} F_{\gamma}\left(x, \alpha_{1}\right)\right\} \\
& = \begin{cases}\left(\gamma+\frac{1}{2}\right)^{2}+F_{\gamma}\left(0, \alpha_{1}\right)=\left(\gamma+\frac{1}{2}\right)^{2} \frac{4+\left(\frac{3}{2}-\gamma\right)^{2}}{2+\left(\frac{3}{2}-\gamma\right)^{2}}, & \text { for } \gamma \leq 1 \\
\left(\gamma+\frac{1}{2}\right)^{2}+\alpha_{1}=\left(\gamma+\frac{1}{2}\right)^{2}+2, & \text { for } \gamma>1\end{cases} \\
& =C_{\gamma}
\end{aligned}
$$

for every solenoidal fields $\boldsymbol{u} \not \equiv \mathbf{0}$. This completes the proof of Theorem 2 .

\section{ApPEndix.}

Here we show that the function $\mathbb{S}^{2} \times \mathbb{S}^{2} \ni\left(\boldsymbol{\sigma}, \boldsymbol{\sigma}^{\prime}\right) \mapsto \log \left(1-\boldsymbol{\sigma} \cdot \boldsymbol{\sigma}^{\prime}\right)$ with $\boldsymbol{\sigma} \neq \boldsymbol{\sigma}^{\prime}$ defines a Green's function of the Laplace-Beltrami operator on $\mathbb{S}^{2}$ :

Lemma 5. For every $f \in C^{\infty}\left(\mathbb{S}^{2}\right)$, let us define the function $G_{f}: \mathbb{S}^{2} \rightarrow \mathbb{R}$ by

$$
G_{f}(\boldsymbol{\sigma})=\int_{\mathbb{S}^{2}} f\left(\boldsymbol{\sigma}^{\prime}\right) \log \left(1-\boldsymbol{\sigma} \cdot \boldsymbol{\sigma}^{\prime}\right) d \sigma^{\prime} \quad \text { for all } \boldsymbol{\sigma} \in \mathbb{S}^{2} .
$$

Then $G_{f}$ is smooth on $\mathbb{S}^{2}$ and satisfies the Poisson-Beltrami equation

$$
\Delta_{\sigma} G_{f}(\boldsymbol{\sigma})=4 \pi f(\boldsymbol{\sigma})-\int_{\mathbb{S}^{2}} f\left(\boldsymbol{\sigma}^{\prime}\right) d \sigma^{\prime} \quad \text { for all } \boldsymbol{\sigma} \in \mathbb{S}^{2} .
$$

proof. We construct the proof in the following steps:

Step 1. First of all, $G_{f}(\boldsymbol{\sigma})$ is well-defined for each $\boldsymbol{\sigma}=(\cos \theta, \sin \theta \cos \varphi, \sin \theta \sin \varphi)$. Indeed, since the matrix $R_{\boldsymbol{\sigma}}=\left(\boldsymbol{\sigma}, \partial_{\theta} \boldsymbol{\sigma}, \partial_{\varphi} \boldsymbol{\sigma}\right)=\left(\boldsymbol{\sigma}, \boldsymbol{e}_{\theta}, \boldsymbol{e}_{\varphi}\right) \in S O(3)$ maps $\boldsymbol{e}_{1}=(1,0,0)$ to $\boldsymbol{\sigma}$ if $\boldsymbol{\sigma} \neq \pm \boldsymbol{e}_{1}$, we have

$$
\begin{gathered}
\int_{\mathbb{S}^{2}} f\left(\boldsymbol{\sigma}^{\prime}\right) \log \left(1-\boldsymbol{\sigma} \cdot \boldsymbol{\sigma}^{\prime}\right) d \sigma^{\prime}=\int_{\mathbb{S}^{2}} f\left(\boldsymbol{\sigma}^{\prime}\right) \log \left(1-\left(R_{\boldsymbol{\sigma}} \boldsymbol{e}_{1}\right) \cdot \boldsymbol{\sigma}^{\prime}\right) d \sigma^{\prime} \\
=\int_{\mathbb{S}^{2}} f\left(R_{\boldsymbol{\sigma}} R_{\boldsymbol{\sigma}}^{-1} \boldsymbol{\sigma}^{\prime}\right) \log \left(1-\boldsymbol{e}_{1} \cdot R_{\boldsymbol{\sigma}}^{-1} \boldsymbol{\sigma}^{\prime}\right) d \sigma^{\prime} \\
=\int_{\mathbb{S}^{2}} f\left(R_{\boldsymbol{\sigma}} \boldsymbol{\sigma}^{\prime}\right) \log \left(1-\boldsymbol{e}_{1} \cdot \boldsymbol{\sigma}^{\prime}\right) d \sigma^{\prime} \\
=\int_{0}^{2 \pi} d \varphi^{\prime} \int_{0}^{\pi} f\left(R_{\boldsymbol{\sigma}} \boldsymbol{\sigma}^{\prime}\right)\left(\log \left(1-\cos \theta^{\prime}\right)\right) \sin \theta^{\prime} d \theta^{\prime}
\end{gathered}
$$


Here the second last equality follows by the change of variables, $R_{\boldsymbol{\sigma}}^{-1} \boldsymbol{\sigma}^{\prime} \mapsto \boldsymbol{\sigma}^{\prime}$. Since the integrand in the last line is bounded on $\mathbb{S}^{2}$, the above integrals are all convergent, which proves $G_{f}(\boldsymbol{\sigma})$ to be well-defined if $\boldsymbol{\sigma} \neq \pm \boldsymbol{e}_{1}$. Moreover, we obtain

$$
G_{f} \in C^{\infty}\left(\mathbb{S}^{2} \backslash\left\{ \pm e_{1}\right\}\right)
$$

from the differentiability of the function $\boldsymbol{\sigma} \mapsto f\left(R_{\boldsymbol{\sigma}} \boldsymbol{\sigma}^{\prime}\right)$. Replacing $f$ by $f \circ R_{\boldsymbol{e}_{2}}$ for the rotation matrix $R_{\boldsymbol{e}_{2}}=\left(\boldsymbol{e}_{2},-\boldsymbol{e}_{1}, \boldsymbol{e}_{3}\right)$, we also have $G_{f \circ R_{\boldsymbol{e}_{2}}}=G_{f} \circ R_{\boldsymbol{e}_{2}} \in C^{\infty}\left(\mathbb{S}^{2} \backslash\left\{ \pm \boldsymbol{e}_{1}\right\}\right)$, which implies $G_{f} \in C^{\infty}\left(\mathbb{S}^{2} \backslash\left\{ \pm R_{\boldsymbol{e}_{2}} \boldsymbol{e}_{1}\right\}\right)=C^{\infty}\left(\mathbb{S}^{2} \backslash\left\{ \pm \boldsymbol{e}_{2}\right\}\right)$. Hence $G_{f} \in C^{\infty}\left(\mathbb{S}^{2}\right)$, as a result.

Step 2. For every $g \in C^{\infty}\left(\mathbb{S}^{2}\right)$, we prove that

$$
F_{g}\left(\boldsymbol{\sigma}^{\prime}\right):=\int_{\mathbb{S}^{2}} \Delta_{\sigma} g(\boldsymbol{\sigma}) \log \left(1-\boldsymbol{\sigma} \cdot \boldsymbol{\sigma}^{\prime}\right) d \sigma=4 \pi g\left(\boldsymbol{\sigma}^{\prime}\right)-\int_{\mathbb{S}^{2}} g(\boldsymbol{\sigma}) d \sigma
$$

for any $\boldsymbol{\sigma}^{\prime} \in \mathbb{S}^{2}$. To do so, we verify the formula

$$
F_{g \circ R}=F_{g} \circ R \quad \text { for every } R \in S O(3) \text {. }
$$

We identify every $g \in C^{\infty}\left(\mathbb{S}^{2}\right)$ with $\widetilde{g} \in C^{\infty}\left(\mathbb{R}^{3} \backslash\{\mathbf{0}\}\right)$ by the relation $\widetilde{g}(\boldsymbol{x})=g(\boldsymbol{x} /|\boldsymbol{x}|)$. Then integration by parts together with the chain rule $\nabla_{\sigma} g(R \boldsymbol{\sigma})=R^{-1} \nabla g(R \boldsymbol{\sigma})$ yields

$$
\begin{aligned}
F_{g \circ R}\left(\boldsymbol{\sigma}^{\prime}\right) & =\int_{\mathbb{S}^{2}}\left(\Delta_{\sigma} g(R \boldsymbol{\sigma})\right) \log \left(1-\boldsymbol{\sigma} \cdot \boldsymbol{\sigma}^{\prime}\right) d \sigma=-\int_{\mathbb{S}^{2}} \nabla_{\sigma} g(R \boldsymbol{\sigma}) \cdot \nabla_{\sigma} \log \left(1-\boldsymbol{\sigma} \cdot \boldsymbol{\sigma}^{\prime}\right) d \sigma \\
& =\int_{\mathbb{S}^{2}}\left(R^{-1} \nabla g(R \boldsymbol{\sigma})\right) \cdot \frac{\boldsymbol{\sigma}^{\prime}-\left(\boldsymbol{\sigma}^{\prime} \cdot \boldsymbol{\sigma}\right) \boldsymbol{\sigma}}{1-\boldsymbol{\sigma} \cdot \boldsymbol{\sigma}^{\prime}} d \sigma=\int_{\mathbb{S}^{2}} \nabla g(R \boldsymbol{\sigma}) \cdot \frac{R \boldsymbol{\sigma}^{\prime}-\left(R \boldsymbol{\sigma}^{\prime} \cdot R \boldsymbol{\sigma}\right) R \boldsymbol{\sigma}}{1-R \boldsymbol{\sigma} \cdot R \boldsymbol{\sigma}^{\prime}} d \sigma \\
& =\int_{\mathbb{S}^{2}} \nabla g(\boldsymbol{\sigma}) \cdot \frac{R \boldsymbol{\sigma}^{\prime}-\left(R \boldsymbol{\sigma}^{\prime} \cdot \boldsymbol{\sigma}\right) \boldsymbol{\sigma}}{1-\boldsymbol{\sigma} \cdot R \boldsymbol{\sigma}^{\prime}} d \sigma=-\int_{\mathbb{S}^{2}} \nabla_{\sigma} g(\boldsymbol{\sigma}) \cdot \nabla_{\sigma} \log \left(1-\boldsymbol{\sigma} \cdot R \boldsymbol{\sigma}^{\prime}\right) d \sigma \\
& =\int_{\mathbb{S}^{2}} \Delta_{\sigma} g(\boldsymbol{\sigma}) \log \left(1-\boldsymbol{\sigma} \cdot R \boldsymbol{\sigma}^{\prime}\right) d \sigma=F_{g}\left(R \boldsymbol{\sigma}^{\prime}\right)
\end{aligned}
$$

where the third and the sixth equality follow from (2.5), and the fifth equality follows by the change of variables $R \boldsymbol{\sigma} \mapsto \boldsymbol{\sigma}$. Hence the proof of $F_{g \circ R}=F_{g} \circ R$ is done. By choosing $R \in S O(3)$ to satisfy $R \boldsymbol{e}_{1}=\boldsymbol{\sigma}^{\prime}$, we see that equation (5.1) is equivalent to

$$
F_{g \circ R}\left(\boldsymbol{e}_{1}\right)=F_{g}\left(R \boldsymbol{e}_{1}\right)=4 \pi g\left(R \boldsymbol{e}_{1}\right)-\int_{\mathbb{S}^{2}} g(R \boldsymbol{\sigma}) d \sigma .
$$

Then replacing $g \circ R$ by $g$ reduces the proof of (5.1) to the special case $\boldsymbol{\sigma}^{\prime}=\boldsymbol{e}_{1}$. This case is verified by integration by parts:

$$
\begin{aligned}
F_{g}\left(\boldsymbol{e}_{1}\right) & =\int_{\mathbb{S}^{2}}\left(\Delta_{\sigma} g(\boldsymbol{\sigma})\right) \log \left(1-\boldsymbol{\sigma} \cdot \boldsymbol{e}_{1}\right) d \sigma=-\int_{\mathbb{S}^{2}} \nabla_{\sigma} g(\boldsymbol{\sigma}) \cdot \nabla_{\sigma} \log (1-\cos \theta) d \sigma \\
& =-\int_{\mathbb{S}^{2}} \partial_{\theta} g(\boldsymbol{\sigma}) \partial_{\theta} \log (1-\cos \theta) d \sigma=-\int_{0}^{2 \pi} d \varphi \int_{0}^{\pi} \partial_{\theta} g(\boldsymbol{\sigma}) \frac{(\sin \theta)^{2}}{1-\cos \theta} d \theta \\
& =-\int_{0}^{2 \pi} d \varphi \int_{0}^{\pi} \partial_{\theta} g(\boldsymbol{\sigma})(1+\cos \theta) d \theta \\
& =-\int_{0}^{2 \pi} d \varphi\left([g(\boldsymbol{\sigma})(1+\cos \theta)]_{\theta=0}^{\theta=\pi}-\int_{0}^{\pi} g(\boldsymbol{\sigma})(-\sin \theta) d \theta\right) \\
& =4 \pi g\left(\boldsymbol{e}_{1}\right)-\int_{\mathbb{S}^{2}} g(\boldsymbol{\sigma}) d \sigma .
\end{aligned}
$$

Thus the proof of (5.1) is done. 
Step 3. By the definition of $G_{f}$, integration of (5.1) with (signed) measure $f\left(\boldsymbol{\sigma}^{\prime}\right) d \sigma^{\prime}$ over $\mathbb{S}^{2}$ yields

$$
\begin{aligned}
\int_{\mathbb{S}^{2}} \Delta_{\sigma} g(\boldsymbol{\sigma}) G_{f}(\boldsymbol{\sigma}) d \sigma & =\iint_{\mathbb{S}^{2} \times \mathbb{S}^{2}} \Delta_{\sigma} g(\boldsymbol{\sigma}) f\left(\boldsymbol{\sigma}^{\prime}\right) \log \left(1-\boldsymbol{\sigma} \cdot \boldsymbol{\sigma}^{\prime}\right) d \sigma d \sigma^{\prime} \\
& =\int_{\mathbb{S}^{2}}\left(4 \pi g\left(\boldsymbol{\sigma}^{\prime}\right)-\int_{\mathbb{S}^{2}} g(\boldsymbol{\sigma}) d \sigma\right) f\left(\boldsymbol{\sigma}^{\prime}\right) d \sigma^{\prime} \\
& =\int_{\mathbb{S}^{2}} g(\boldsymbol{\sigma})\left(4 \pi f(\boldsymbol{\sigma})-\int_{\mathbb{S}^{2}} f\left(\boldsymbol{\sigma}^{\prime}\right) d \sigma^{\prime}\right) d \sigma
\end{aligned}
$$

On the other hand, since $g$ and $G_{f}$ are smooth, the self-adjointness of $\Delta_{\sigma}$ in $L^{2}\left(\mathbb{S}^{2}\right)$ gives

$$
\int_{\mathbb{S}^{2}} g(\boldsymbol{\sigma}) \Delta_{\sigma} G_{f}(\boldsymbol{\sigma}) d \sigma=\int_{\mathbb{S}^{2}} \Delta_{\sigma} g(\boldsymbol{\sigma}) G_{f}(\boldsymbol{\sigma}) d \sigma
$$

Hence combining the two results yields

$$
\int_{\mathbb{S}^{2}} g(\boldsymbol{\sigma}) \Delta_{\sigma} G_{f}(\boldsymbol{\sigma}) d \sigma=\int_{\mathbb{S}^{2}} g(\boldsymbol{\sigma})\left(4 \pi f(\boldsymbol{\sigma})-\int_{\mathbb{S}^{2}} f\left(\boldsymbol{\sigma}^{\prime}\right) d \sigma^{\prime}\right) d \sigma
$$

Since the choice of $g \in C^{\infty}\left(\mathbb{S}^{2}\right)$ is arbitrary, the two integrands in the both sides must coincide, which proves the desired equation.

Acknowledgments. The author would like to thank Prof. Futoshi Takahashi (Osaka City University) for his helpful advice and encouragement in this study. Additionally, this work was partly supported by Osaka City University Advanced Mathematical Institute (MEXT Joint Usage/Research Center on Mathematics and Theoretical Physics).

\section{REFERENCES}

[1] O. Costin and V. Maz'ya: Sharp Hardy-Leray inequality for axisymmetric divergence-free fields, Calc. Var. Partial Differ. Equ. 32 (2008), no. 4, 523-532.

[2] R. Courant and D. Hilbert: Methoden der Mathematischen Physik I, ch. V, Springer, Berlin, Heidelberg, 1924 (German).

[3] N. Hamamoto: Sharp Rellich-Leray inequality for axisymmetric divergence-free vector fields, Calc. Var. Partial Differ. Equ. 58 (2019), no. 4.

[4] N. Hamamoto and F. Takahashi: Sharp Hardy-Leray and Rellich-Leray inequalities for curl-free vector fields, arXiv:1808.09614v1 [math.AP] (2018).

[5] N. Hamamoto and F. Takahashi : Sharp Hardy-Leray inequality for three-dimensional solenoidal fields with axisymmetric swirl, arXiv:1904.01338 [math.AP].

[6] G. H. Hardy: Note on a theorem of Hilbert, Math. Z. 6 (1920), no. 3-4, 314-317.

[7] Y. Kimura and H. Okamoto: Vortex motion on a sphere, J. Phys. Soc. Japan 56 (1987), no. 12, 4203-4206

[8] J. Leray: Étude de diverses équations intégrales non linéaires et de quelques problèmes que pose l'Hydrodynamique, J. Math. Pures Appl., 12 (1933), 1-82.

[9] B. Sri Padmavati and T. Amaranath: A note on decomposition of solenoidal fields, Appl. Math. Lett. 15 (2002), no. 7, 803-805.

Department of Mathematics, Osaka City University 3-3-138 Sugimoto, Sumiyoshi-ku, Osaka 558-8585, JAPAN

E-mail address: yhjyoe@yahoo.co.jp (N. Hamamoto) 